\title{
A Contingent Cost Analysis of Alternative Manufacturing Information Systems
}

\author{
R.B. COOPER \\ Graduate School of Business Administration, The University of \\ Michigan, Ann Arbor, Michigan 48109, USA
}

\begin{abstract}
The evaluation of alternative management information systems can aid systems analysts determine appropriate information support for managers, aid managers to become informed participants in the system design process, and aid the planning of information systems in response to changes in the firm. Synthesizing microeconomic and contingency theories, this paper performs a cost analysis of two popular manufacturing information systems. The relative cost-effectiveness of these two systems is hypothesized and then validated using a random survey of manufacturing firms across the USA. A logistical regression of the data provides estimates of the relative information system cost-effectiveness in various manufacturing contexts.
\end{abstract}

I would like to thank Burt Swanson and Dennis Severance for their helpful comments on earlier drafts of this paper.

\footnotetext{
${ }^{1}$ The American Production and Inventory Control Society defines production and inventory management as "The function of directing or regulating the orderly movement of goods through the entire manufacturing cycle from the requisitioning of raw materials to the delivery of the finished product to meet the objectives of customer service, minimum inventory investment, and maximum manufacturing efficiency MOORE, F.C. AND HENDRICK, T.H. (1980). Production/Operations Management, 8th edn. Homewood, Ill: Richard D. Irwin.

${ }^{2}$ AGGARWAL, S.C. (1985). MRP, JIT, OPT, FMS? Making sense of production operations systems. Harvard Business Review, September-October, 8-12.

${ }^{3}$ For example, wiGitr, o.w. (1974). Production and Inventory Management in the Computer Age, Boston, Mass.: Cahners Books.
}

\section{Introduction}

The evaluation of alternative management information systems (MIS) is important from three perspectives; that of the information resource manager, that of the systems analyst, and that of the user. Such evaluations help information resource managers plan future MIS requirements, help systems analysts determine the appropriate MIS support for current management decision-making, and help users become informed participants in the system development process. This paper uses microeconomic production theory within a contingency theoretic framework to evaluate two widely used manufacturing MIS. The results of this evaluation provide interesting insights into the relative cost-effectiveness of Material Requirements Planning and Reorder-Point MIS in various manufacturing contexts.

The increasing pressure upon US manufacturing firms to make their processes more efficient and effective makes it appropriate to place this MIS evaluation in a manufacturing environment. An important factor for improving these manufacturing processes is production and inventory management (PIM), ${ }^{1}$ and a variety of PIM information systems such as Material Requirements Planning (MRP) and Optimized Production Technology (OPT) have thus been developed to replace the traditional Reorder-Point based MIS. Of these new information systems, MRP has had the most extensive implementation. ${ }^{2}$ This paper focuses upon MRP and its replacement of traditional Reorder-Point based systems because, although extensively implemented, MRP has had a very significant history of failure. For example, although MRP has been acclaimed as making traditional Reorder-Point based systems obsolete, ${ }^{3}$ as Plossl notes, "never . . . has so much been proclaimed and expected and so little actually delivered'. ${ }^{4}$ While MRP is still highly promoted, descriptions of MRP failure abound in the literature. ${ }^{5}$

Most diagnoses indicate a variety of reasons for MRP failure, including resistance to change, ${ }^{6}$ lack of software integration, ${ }^{7}$ lack of management understanding and commitment to MRP, ${ }^{8}$ and inaccurate work-in-process inventory accounting. ${ }^{9}$ It is hypothesized here that an important cause of MRP failure stems from attempts to implement MRP in inappropriate contexts. That is, even if there is no resistance, the software is appropriately designed and implemented, management is totally committed, and data are current and accurate, MRP will fail in 
${ }^{4}$ PLossi, , G.w. (1980). MRP yesterday, today, and tomorrow. Production and Inventory Management, third quarter, 1-9. ${ }^{5}$ For example, PLossi, ci.w. (1985). Production and Inventory Control: Principles and Techniques. Now Jersey: Prentico Hall. wikillt, 0.w. (1982). The Executive's Guide to Successful MRPII. Englewood Cliffs: Prentice Hall.

"WHITF, F.M. (1980). Implementing an MRP system using the Lewin-Schein theory of change. Production and Inventory Management, first quarter, 1-12.

BAHI., H.C. AND RITZMAN, 1..P. (1983). An empirical investigation of different stratcgies for material requirements planning. Journal of Operations Management, 3. 67-79.

"130XX, D.B. (1979). MRP-Selling a dead horse (or how to convince management to invest in MRP). Production and Inventory Management, second quarter, 49-62.

"FRFNC11, R.L. (1980). Accurate work in process inventory-a critical MRP system requirement. Production and Inventory Management, first quarter, 17-22.

I"MORECROFT, J.D.w. (1983). A systems perspective on material requirements planning. Decision Sciences, 14, 1-18.

${ }^{11}$ MILES, R. (1980). Macro Organizational Behavior, pp. 248-278. Santa Monica: Goodyear Publishing.

${ }^{12}$ For example, IIANNAN, M. AND FRFEMAN J. (1977). Population coology of organization. American Journal of Sociology, 82 929-964. Alidrich, 11.1. (1979). Organizations and Environments. Englewood Cliff: Prentice Hall. mokelvi: Y, B. (1983). Organizational Systematics: Taxonomy, Evolution, Classification, Berkeley: University of California Press.

${ }^{13}$ VAN DE VEN, A.H. ANI) IDRA ZIN, R. (1985). The concept of fit in contingency theory. In Research in Organizational Behavior, L.L. Cummings and B.M. Straw eds. Vol 7 . Greenwich, Connecticut: JAI Press.

${ }^{14}$ COOPER, R.B. (1985). Identifying appropriate MIS/DSS support: A cost analysis approach. Proceedings of the Sixth International Conference on Information Systems. Indianapolis, pp. 89-104. certain manufacturing contexts. (This vicw is in accord with Morecroft's simulation studies. ${ }^{10}$ )

Since MRP failure is relative, it is defined here as resulting in poorer PIM decisions than would traditional Reorder-Point MIS in a given context. This implies that Reorder-Point systems will also fail, i.e., do worse than MRP, in other manufacturing contexts. It is of interest, then, to determine which of the two MISs is appropriate for a given context. This will enable system analysts and users to choose an appropriate MIS for the current context, and will help information resource managers and users plan an evolving MIS to match an evolving production context.

This focus upon the interaction of context and MIS with decisionmaking effectiveness provides the link to contingency theory. As noted by Miles, ${ }^{11}$ contingency theory can be useful in determining the effectiveness of alternative decision-making structures in various contexts. Here, decision-making structure is reflected by the structure of the information systems employed in the decision process. According to contingency theory, decision-making effectiveness can be identified by the observed correlation of specific decision-making structures with specific contexts. There is thus an assumed causal relationship in which decision-making effectiveness is determined by the interaction of structure and context. This assumption is supported by a natural selection argument. ${ }^{12}$ That is, in the long run, decision structures which are not effective in specific contexts will be competed away.

In addition to the use of structure-context correlations to infer effectiveness, there are lines of contingency theory research which directly measure the impact upon effectiveness of specific structures within certain contexts. These research lines have been referred to by Van de Ven and Drazin ${ }^{1.3}$ as the interaction approach and the systems approach. The systems approach is the more general of the two, and allows for a compensatory relationship between structural components within a context. Thus, different decision-making structures can result in equally effective decisions in a specific context.

Both the structure-context correlations approach, which infers decision-making effectiveness, and the systems approach, which directly determines the impact of structure and context upon decision-making effectiveness, are employed in this paper. The systems approach provides a framework which guides a cost analysis of the alternative manufacturing MIS. The structure-context correlations approach is then employed to validate the cost analysis results.

A brief introduction to cost analysis and its integration with contingency theory is provided next (a more detailed description of cost analysis is provided by Cooper ${ }^{14}$ ). This is followed by its application to the MRP versus Reorder-Point evaluation.

\section{Introduction to cost analysis}

Cost analysis is built upon microeconomic production theory. However, the focus is upon the production of decisions rather than physical units. This production can be modelled by linking the use of certain physical resources (computer software, managers, communications devices, etc.) to decisions. As with physical production, a transformation of raw materials occurs. The raw material of decision production is information, transformed from its initial state (data) to its final state (decisions). 
The output of decision production (i.e., decisions) is treated as one of many inputs to a firm's production system. For example, in the manufacture of cars, manufacturing planning and control decisions are considered a class of input along with raw materials, equipment and non-management labour. The measurement of decisions occurs along many dimensions, which generally fall within the intuitive concept of decision quality. (See Cooper ${ }^{15}$ for a detailed discussion of decision production and decision quality.)

\section{Cost curve derivation}

Within this view, decision quality is a function of the decision-maker(s), the MIS, and the decision context. For a given decision-maker and a given MIS, one would generally expect that the quality of decisions will change as the decision context changes. This is in accord with contingency theory's notion of decision structure-context fit. For example, compare a firm which produces one product to stock (say continuous production of a chair) with a firm which produces anything the customer orders (e.g., a metal-working job shop producing to customer specifications). In the first instance, a relatively simple MIS (e.g., a 'two-bin' Reorder-Point system) and a relatively unsophisticated PIM decision-maker could provide decisions of adequate quality. However, if the same MIS and decision-maker were used in the second firm, they would be overwhelmed by its complexity, which would result in decisions leading to excessive production cost and unmet customer orders.

The above discussion focused on the effect of decision context changes upon decision quality. By holding decision quality constant, effects upon the decision-maker and MIS required by changes in the decision-context can be addressed. For example, in considering the second firm described above, what would it take to make good quality decisions (i.e., decisions which do not lead to excessive production cost and unmet customer orders) if the formal information system were limited to the simple two-bin Reorder-Point system? One strategy might be to hire staff personnel and a more sophisticated decision-maker to make up for the inadequacy of the MIS and the decision-maker. This new group would then spend time gathering data - such as machine availability and current product status - not provided by the Reorder-Point system. The more sophisticated decision-maker would then use these data to help plan more efficient and effective production. A major consequence of this strategy would be increased cost: the more sophisticated decision-maker and the staff personnel would be more expensive than the unsophisticated decision-maker they replaced. This effect of decision context changes upon total decision-making cost is depicted in Figure 1, where the horizontal axis represents the change from a relatively simple manufacturing environment (producing one type of chair to stock) to a relatively complex environment (a job shop producing to various customer specifications).

This example has illustrated an assumption of cost analysis. That is, the quality of decisions can generally be maintained in the face of changing contexts by changing the characteristics of the information system and/or the decision-maker(s). This is in accord with the equifinality proposition of contingency theory. ${ }^{16}$ In addition, changing contexts can generally be defined in terms of continuous dimensions, such as manufacturing context complexity, which facilitates the drawing 


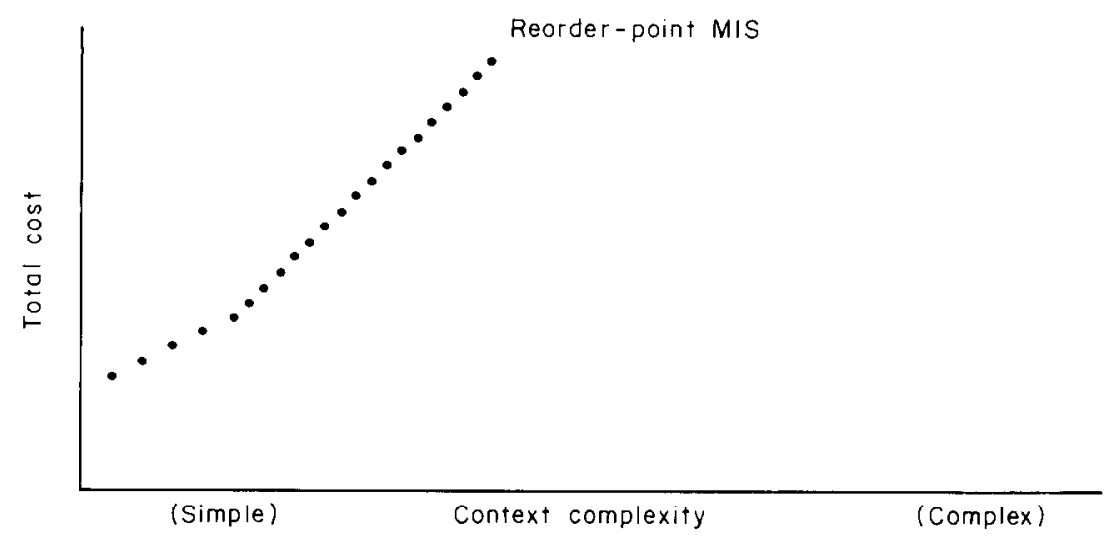

Figure 1. Total cost curve for one MIS

of total decision-making cost curves.

Reconsider the above scenario, replacing the very simple ReorderPoint MIS with a more sophisticated MRP system. MRP proponents typically claim that MRP would work as well as the Reorder-Point system in the simple context (that of the first firm), and out-perform the Reorder-Point system in the complex context. If this is true, then the MRP system provides better (more useful, more comprehensive) information in the complex context; the result, when compared to Reorder-Point, is that less staff expense is required to maintain decision quality. This differing effect of context complexity upon decisionmaking cost for the two MISs is illustrated in Figure 2. Note that MRP's initial cost is higher than that of Reorder-Point. This is due to the typically higher implementation and maintenance costs associated with MRP systems.

The cost curves which hold decision quality constant in Figure 2 form the basis of cost analysis comparisons of alternative MIS. Reorder-Point systems are illustrated as more cost-effective for less complex contexts, while MRP is more cost-effective for more complex contexts. Based upon the assumptions of cost analysis, this would be true for reasonable levels of decision quality.

Cost-effectiveness, then, is the contingency theoretic effectiveness

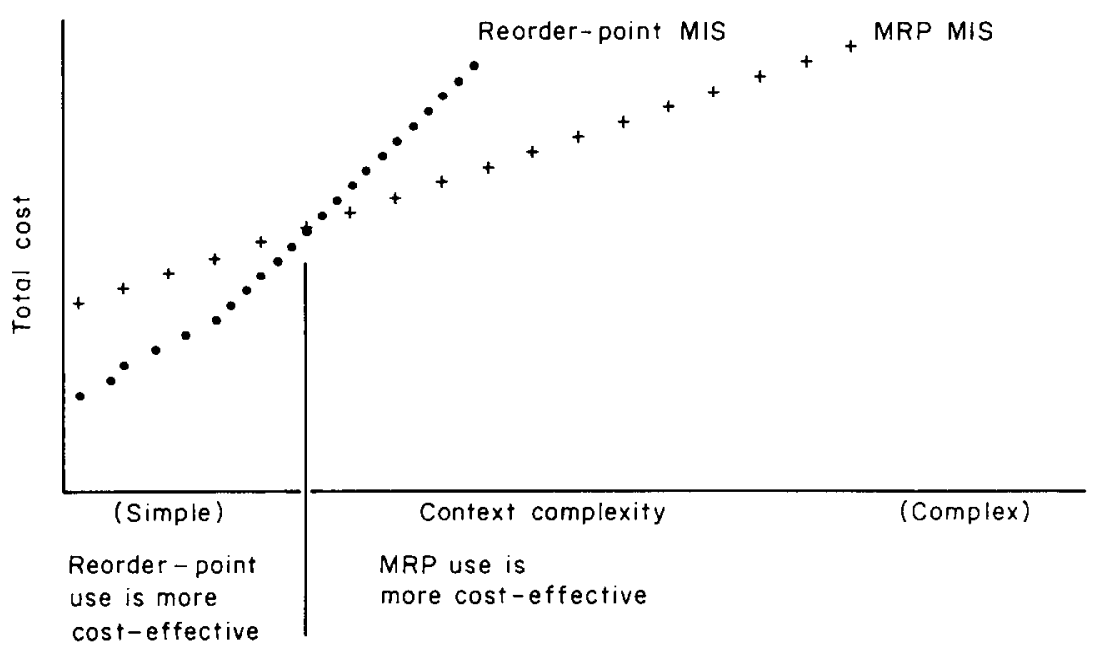

Figure 2. Hypothetical example of total cost curves for two alternative MISs 
measure. Decision structure, as reflected by the use of alternative MIS, interacts with the manufacturing context resulting in more or less cost-effective decision-making (at a constant decision quality). Although the effect of a single - rather general - contextual dimension (complexity) was examined in the example above, subsequent analysis of MRP versus Reorder-Point based decision-making will employ multiple specific dimensions. Similarly, although a single general dimension of decision structure (sophistication) was examined, subsequent analysis will employ multiple specific decision-structure dimensions.

It was not the purpose of this section to argue that the cost-curve relationships in Figure 2 are correct. In fact, the analysis below will show that the illustrated relationships are not correct. The reason they were used for discussion purposes is because they reflect popular opinion concerning MRP versus Reorder-Point effectiveness. The next sections use the cost analysis methodology to hypothesize actual MRP versus Reorder-Point cost-curve relationships and then test these hypotheses.

\section{MIS and contextual dimensions}

As applied to this alternative MIS evaluation, the cost analysis methodology consists of the following steps:

1. Identify the MIS dimensions on which the alternative MISs differ significantly. In the above hypothetical example, the 'two-bin' Reorder-Point system and the MRP system differed in terms of two MIS dimensions: MIS sophistication and MIS implementation and maintenance costs.

2. Identify the contextual dimensions which interact with the above MIS dimensions resulting in changes in decision-making cost. A dimension of context complexity was used above.

3. Develop hypotheses about alternative MIS cost-effectiveness based upon interactions between the above MIS dimensions and contextual dimensions. The Reorder-Point system was hypothesized to be more cost-effective in less complex contexts and the MRP system was hypothesized to be more cost-effective in more complex contexts.

4. Gather empirical evidence to test the hypothesized effects. This was not illustrated above.

5. Draw conclusions concerning the cost-effectiveness of the alternative MIS. This was not illustrated above.

This section is devoted to the first two cost analysis steps. MIS dimensions and contextual dimensions appropriate for the analysis of MRP versus Reorder-Point systems are determined from the production literature. The appropriateness of these dimensions is based on their effect upon the cost of decision-making. This cost can be affected either directly via MIS implementation and maintenance costs, or indirectly via the impact upon decision quality. As illustrated above, the indirect effect occurs when changes in a contextual dimension impact the decision-making usefulness of an MIS dimension. In order to maintain a constant decision quality, this change in MIS usefulness must be offset by changes in the amount and type of other decision-making resources, which result in changes in decision-making expenses. For example, problem duration (the time available for the solution of a problem) 
probably affects the usefulness of fast versus slow MIS response time: if a problem can be solved in a week, a real-time MIS may be no more useful than a batch MIS; however, if a problem must be solved in one hour, the batch MIS may be of little use, and supplemental decisionmaking resources would have to be employed in order to maintain good quality decisions. If the alternative MISs differ significantly in terms of response time, then response time is an appropriate MIS dimension, and problem duration is an appropriate contextual dimension.

To begin this selection process, some MIS dimensions are enumerated below:

1. Response time - the time required for an MIS to provide requested information. For example, batch versus real-time.

2. Model structure - the MIS assumptions and information describing causal relationships, appropriate variables and decision-maker preferences.

3. Access restrictions - the difficulty in getting use of the computer (MIS) facilities. For example, scheduled periodic access versus unscheduled ad hoc access.

4. Decision-making focus - the emphasis of the MIS in support of decision-making. This ranges from helping define or structure problems to actually making the decisions.

5. Implementation and maintenance costs - the costs reflecting the needs for computerization, organizational and procedural changes, and software and data maintenance.

By concentrating on MIS dimensions upon which the alternative MISs differ significantly, the cost analysis evaluation effort can be reduced. MRP and Reorder-Point MIS are, in fact, very similar. Response time is typically daily or weekly, access restrictions are typically periodic (e.g., weekly) and scheduled, and the decision-making focus tends toward decision-making rather than decision-structuring. MIS dimensions on which MRP most differs from Reorder-Point are model structure and implementation and maintenance costs. For example, MRP model structure is oriented toward dependent inventory item demand, where requirements for sub-components are derived from the requirements for components. In contrast, Reorder-Point model structure is orientated toward independent inventory item demand, where requirements for each item are determined independently. In addition. MRP implementation and maintenance costs are typically more than those for Reorder-Point, and tend to be more sensitive to the complexity of the production process due to extensive data and model maintenance.

With the identification of these two MIS dimensions, analysis continues to get a more precise notion of how MRP differs from Reorder-Point along each dimension. This is described first for the model structure dimension, and then for the implementation and maintenance cost dimension.

The importance of the model structure dimension for differentiating MRP from Rcorder-Point is due in large part to its implications for the ability of an MIS model to fit within its context. To this end, a model's assumptions can be compared with the context, and the extent to which the assumptions are violated can form a basis for cost analysis evaluation. The notion here is that an MIS becomes less useful as the underlying assumptions of its model(s) become increasingly violated. Since cost analysis focuses upon differences between alternative MIS, 
assumptions not shared by both MRP and Reorder-Point are of major interest. For example, since the assumptions of constant manufacturing/ purchase lead time and constant costs are shared by MRP and Reorder-Point, they are ignored here. The PIM literature is thus reviewed ${ }^{17}$ to find typical assumptions which are not common to both MRP and Reorder-Point MIS. The following unshared model assumptions were identified:

1. MRP:

(a) Finished goods requirements are deterministic.

(b) Processes are independent (no requirements for 'mating' of processes).

2. Reorder-Point:

(a) Average inventory item usage is continuous and constant over time with random deviations described by a constant distribution.

(b) Inventory items are independent: the demand or replenishment of one does not affect that of another.

Thus, the model structure dimension is more precisely defined in terms of two MRP assumptions, two Reorder-Point assumptions.

A more precise definition of the implementation and maintenance costs dimension is addressed next. Costs identified by this dimension reflect the needs for computerization, organizational and procedural changes, and software and data maintenance. When compared to Reorder-Point, MRP is initially more expensive due to extensive computer and organizational changes, and its maintenance costs are much more sensitive to the complexity of the production process due to extensive data and model maintenance requirements. Thus, MRP implementation and maintenance costs are expected to increase more with increased production process complexity than Reorder-Point implementation and maintenance costs. All the costs indicated in the MIS dimension should thus be used to help differentiate MRP from Reorder-Point.

The second cost analysis step consists of identifying manufacturing contextual dimensions which significantly interact with these MIS dimensions to affect decision-making cost. Contextual dimensions which interact with model structure (i.e., those that violate MRP and Reorder-Point assumptions) are described first. Two contextual dimensions which can be easily identified and tend to have this interaction effect are a firm's manufacturing method and the marketing strategy. ${ }^{18}$

${ }^{17}$ Buffa, E.S. (1980). Modern Production/ Operations Management. 6th edn., Chichester: John Wiley \& Sons. BUF:A, E.S. ANI MIll. ere, J.G. (1979). ProductionInventory Systems Planning and Control. 3rd edn., Homewood, Ill.: Richard D. Irwin. ORLIICKY, J. (1975). Material Requirements Planning. New York: McGrawHill. PlossL, G.w. (1973). Manufacturing Control, the Last Frontier for Profits. Reston, VA: Reston Publishing Co. sll,ver. F.A. (1981). Operations research in inventory management: A review and critique. Operations Research, 29, 628-645.

18 BUfFer and mili.er, op. cit., Ref 17.

19 VANDEMARK, R.L. (1964). Production Control Techniques. Grand Rapids: The Gilson Press. voris, w. (1961). Production Control. Homewood, III.: Richard D. Irwin.
Manufacturing method depicts characteristics of production itself, and can be measured along a continuum ranging from continuous (or assembly or repetitive) to intermittent (or job shop). Marketing strategy can also be measured along a continuum, ranging from all products made-to-stock to all products made-to-order. A study of the production and inventory control literature ${ }^{19}$ reveals the following relationships between these contextual dimension measures and MRP and ReorderPoint assumption violation:

1. MRP:

(a) A significant violation of deterministic finished goods requirements is typically associated with intermittent manufacturing and with make-to-order contexts. This is due, for example, to uncertainty associated with the variety of custom designs and the unreliability of sales forecasts, respectively. 
(b) No relationship between either manufacturing method or marketing strategy and process independence could be found.

2. Reorder-Point:

(a) A significant violation of continuous and constant inventory item usage is typically found in continuous manufacturing and in make-to-stock contexts. This is due, for example, to the more stable and forecastable market which allows systematic production variations. These variations typically occur within Reorder-Point review cycles and thus do not result in Reorder-Point changes.

(b) No relationship between either manufacturing method or marketing strategy and inventory item independence could be found.

Thus, measurements along a firm's manufacturing method and marketing strategy contextual dimensions enable an evaluation of violation for one MRP and one Reorder-Point assumption. Using general descriptions of firm activities, it would be hard to measure the degree of process independence. However, a good estimate of the inventory item independence contextual dimension - which interacts with Reorder-Point's assumption of inventory item independence - can be ascertained by determining the average number of bill-of-materials levels required for production. In fact, this measure is typically used for this purpose, and is thus employed here as a measure of inventory item independence.

Contextual dimensions which interact with the MRP and ReorderPoint implementation and maintenance costs are also of interest. Differences between these costs for MRP and Reorder-Point were described earlier as dependent in large part upon the complexity of the production process. Two readily identifiable measures for this contextual dimension are the average number of parts (components, etc.) per bill-of-material level and the average number of parts (components, etc.) per finished good.

The first two cost analysis steps in evaluating MRP versus ReorderPoint MIS have been described. More precise notions of two MIS dimensions (model structure and implementation and maintenance costs) have been determined and paired with measures for four contextual dimensions (marketing strategy, manufacturing method, inventory item independence, and production process complexity). 'The next section addresses the third cost analysis step.

\section{Alternative MIS cost-effectiveness hypotheses}

This section describes the third cost analysis step: the development of hypotheses about alternative MIS cost-effectiveness based upon interactions between MIS and contextual dimensions. There are two sub-steps which comprise this third step. Using earlier discussions which paired MIS dimensions with contextual dimensions, the first sub-step identifies the resulting decision cost-curve shapes. The second sub-step then develops hypotheses concerning the cost-effectiveness of the alternative MIS based upon these decision cost curves.

Decision cost-curve shapes representing the interaction of MIS model assumptions with the associated contextual dimension measures are 
relatively straightforward. As mentioned earlier, basic to this interaction is the notion that an MIS becomes less useful as the underlying assumptions of its model(s) become increasingly violated. That is, a model is less useful if it assumes a reality different from that in which it is employed. Given the cost analysis framework, this decrease in MIS usefulness can be offset by increasing expenditures on other decisionmaking resources, leading to cost curves similar to that in Figure 1. For example, the Reorder-Point inventory item independence assumption is paired with the inventory item independence contextual dimension: the independence assumption is increasingly violated as the average number of bill-of-material levels increase. With the average number of bill-of-material levels on the horizontal axis, and total decision-making cost on the vertical axis, the cost curve for Reorder-Point MIS is expected to rise (as in Figure 1); since no MRP assumption violation occurs along this axis, the cost curve for MRP MIS is not expected to be significantly affected.

Deterministic finished goods requirements paired with the marketing strategy contextual dimension is an example of MRP assumption violation. In this case, the marketing strategy is on the horizontal axis, and ranges from pure make-to-stock through various combinations of make-to-stock and make-to-order, and ends with pure make-to-order. The MRP assumption of deterministic finished goods requirements is increasingly violated along this horizontal axis. The result is a rising MRP cost curve similar to those illustrated in Figure 3 . Note that the Reorder-Point assumption of continuous inventory item usage is also violated along the marketing strategy dimension, but in an opposite manner. Thus, when the Reorder-Point cost curve is plotted in the same graph, it decreases from make-to-stock to makc-to-order. This is illustrated in Figure 3, where MRP is identified as more cost-effective in make-to-stock contexts and Reorder-Point is identified as more cost-effective in make-to-order contexts.

The decision cost-curve shapes depicting the interaction of MIS implementation and maintenance costs with production process complexity are similar to that in Figure 1 for both contextual dimension measures. However, the two contextual dimension measures are expected to affect MRP total decision-making cost much more

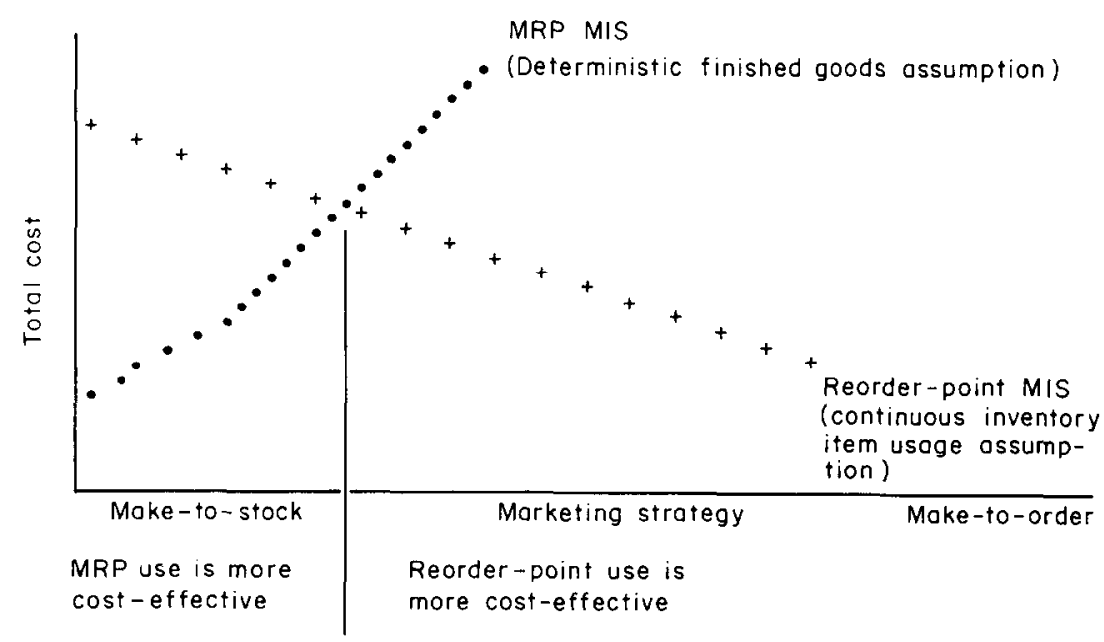

Figure 3. Hypothesized cost curves for MRP and Reorder-Point MIS along the marketing strategy contextual dimension 
significantly than that of Reorder-Point. Thus, for example, with average number of parts per finished good on the horizontal axis, and total decision-making cost on the vertical axis, the cost curve for MRP MIS is expected to rise much more significantly than the cost curve for Reorder-Point MIS.

Each of the four contextual dimensions mentioned above form one axis in a five-dimensional decision-making cost space. (The fifth dimension is total decision-making cost.) Plotting the decision-making cost behavior described above in this space results in two cost surfaces: one for MRP and one for Reorder-Point. An examination of these cost surfaces relative to each contextual dimension axis results in the following hypotheses. MRP tends to be more cost-cffective than Reorder-Point in:

$\mathrm{H}_{1}$ Continuous (assembly, repetitive) manufacturing method than in intermittent (job shop) manufacturing method contexts.

$\mathrm{H}_{2}$ Make-to-stock marketing strategy contexts than in make-to-order marketing strategy contexts.

$\mathrm{H}_{3}$ Contexts which have a high number of bill-of-materials levels.

$\mathrm{H}_{4 \mathrm{a}}$ Contexts in which the average number of parts, etc. per bill-ofmaterial level are low.

$\mathrm{H}_{4 \mathrm{~b}}$ Contexts in which the average number of parts, etc. per finished good are low.

Hypotheses 1, 2 and 3 are based upon manufacturing method, marketing strategy and inventory item independence contextual dimension's interaction with MRP and Reorder-Point model assumptions. Hypotheses $4 \mathrm{a}$ and $4 \mathrm{~b}$ are based upon the production process complexity contextual dimension's interaction with MIS implementation and maintenance costs.

Up to this point, the systems approach of contingency theory has been used as a framework to develop expectations of the impact upon decision cost-effectiveness of alternative MIS dimensions within various contextual dimensions. The link to contingency theory now shifts to a more traditional approach: the inference of MRP cost-effectiveness based upon its use in specific contexts. As mentioned earlier, the assumption underlying such an inference is one of natural selection; thus, MRP use will tend to be observed only in contexts where MRP is cost-effective. Note that this view ties neatly to the microeconomic literature, where rational behaviour can be viewed as an artifact of competition - firms behaving irrationally will not last in the long run rather than a description of specific decision-making ability. ${ }^{211} \mathrm{~A}$ study of current manufacturing MIS use is thus undertaken. This study is described next.

\section{A study of current manufacturing MIS use}

If the above hypotheses are correct, then rational managers will tend to employ MRP in continuous, make-to-stock contexts with products having a high number of bill-of-material levels, a low number of parts per bill-of-material level, and a low number of parts per finished good. This implies that managers who try MRP in other contexts will tend to

Eot AICHAN, A. (1963). As quoted in $A$ Behavioral Theory of the Firm (R.M. Cyert and J.G. March, eds), p. 13. Englewood Cliffs: Prentice-Hall. be confronted with excessive decision-making costs, and would thus be encouraged to return to Reorder-Point MIS. Based upon this rationale, the fourth cost analysis step involves a survey of current MRP use, 
which can serve to support or deny any or all of the hypotheses. That is, if MRP is typically used in the proposed contexts, and not typically used in the other contexts, then the hypotheses are supported based upon rational (or, competitively advantages) management trade-offs.

\section{The survey}

Survey respondents consisted of a random sample of the American Production and Inventory Control Society members across the USA. ${ }^{21}$ A telephone interview approach was chosen to reduce confusion over terminology and to encourage a high response rate. Production managers and staff who wanted time to look data up were called back. The response rate was 97 per cent of contacted applicable members. Of the 100 -member random sample, 36 were eliminated by logistical problems or because they failed to meet survey requirements (c.g., they were teachers rather than production managers or staff). Of the remaining members, only two refused to answer the survey. Hence, the results are based upon production managers and staff representing a random sample of 62 manufacturing facilities in the USA.

Respondents were asked about their firm's manufacturing method, marketing strategy, average bill-of-material levels, etc. In addition, they were asked what kind of manufacturing MIS was used. If they were using MRP, they were also asked to rate the system using the classification scheme used by Anderson et al. ${ }^{22}$ This scheme ranges from 'A' MRP, which is fully integrated into the production planning process, to 'D' MRP, in which the system exists mainly in data processing and is of little benefit to production managers. It should be noted that all firms included in the survey which were not using MRP were using a traditional Reorder-Point-based MIS.

\section{The results}

Data were coded in the following manner. Manufacturing method was coded 0 for continuous and 1 for intermittent. Manufacturing strategy was coded 0 for make-to-stock and 1 for make-to-order. The average number of bill-of-material levels, the average number of parts, etc. per bill-of-material level, and the average number of parts, etc. per finished good were all coded as dummy variables, with 0 for values less than their medians, and 1 for values greater than their medians. Median values for these variables are 4.5 bill-of-material levels, 6.5 parts per bill-ofmaterial level, and 24 parts per finished good. This approach was chosen to reduce the precision of the variables commensurate with the

'Membership exceeds 50 000. I would like to thank Henry F. Sander, Executive Director of the American Production and Inventory Control Society, for his cooperation in providing the survey sample used in this paper.

${ }_{22}^{2}$ ANDERSON, J.C, SCHROLDLE, R.G., TUPY, S.E. AND WHITF, E.M. (1981). Material Requirements Planning: A Study of Implementation and Practice. Falls Church, Virginia: American Production and Inventory Control Society.

2.3 BAI.L, C.A. AND TSCHOHGG., A.E. (1982). The decision to establish a forcign bank branch or subsidiary: an application of binary classification procedures. Journal of Financial and Quantitative Analysis, 17 , 411-424. Ineasurement error associated with their estimates. The MRP use variable was coded 1 for ' $D$ ' category use or Reorder-Point use, and 2 for category 'C' or above MRP use. Thus, MRP use is defined, at lcast, as employment of the MIS as an order-launching system.

Because of dichotomous dependent and independent variables, a logistic regression model (logit) is used to test the hypotheses. ${ }^{23}$ Table 1 illustrates the results: the overall model is highly significant (alpha $=$ 0.0025 ) and all hypotheses are supported at alpha less than or equal to 0.07. Note that $\mathrm{H}_{4 \mathrm{~b}}$ (average number of parts, etc. per finished good) is not in the logit model. This is due to the very high collinearity with $\mathbf{H}_{4 ; \mathrm{a}}$ (average number of parts, etc. per bill-of-material level). The Pearson Correlation cocfficient between the two variables is 0.88 , significant at alpha less than 0.01 . Thus, the average number of parts, etc. per bill-of-material level is a good representative of hypothesis 4 . Note, 
Table 1. Logistic regression for MRP use $=C$ or $B$ or $A$

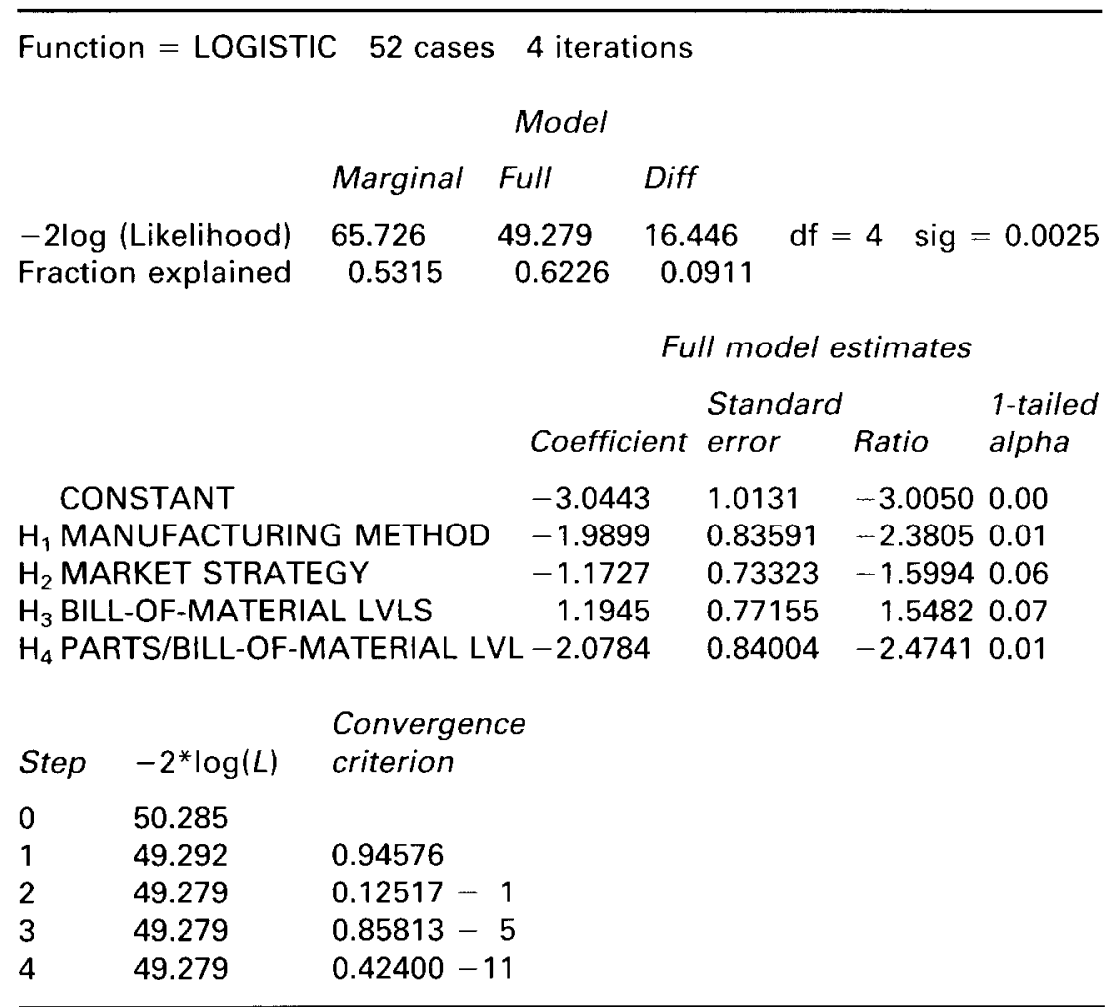

also, that only 52 cases were used rather than the 62 available from the sample. This is due to ambiguity regarding marketing strategy: some firms made to stock and to order. Since a dichotomous marketing strategy variable was used, only firms which manufactured 80 per cent or more of their product exclusively to stock or exclusively to order were included. Ten firms were eliminated for this reason.

The definition of MRP use employed here diverges somewhat from that desired by MRP proponents. Rather than considering order launching (category $\mathrm{C}$ ) as successful MRP use, MRP proponents suggest that MRP is not being used properly until it is more fully integrated into production planning, i.e., category $\mathrm{A}$ or $\mathrm{B}$. The effect of changing the MRP use definition in this manner was tested, and, as illustrated in Table 2, the overall model was not significant (alpha $=0.4725$ ). In this insignificant model, MRP use was coded 1 for category $C$ or D MRP use or Recorder-Point use and coded 2 for category A or B MRP use.

In summary, MRP tends to be used in the hypothesized manufacturing contexts, which are characterized by:

1. Continuous (assembly, repetitive) manufacturing method.

2. Make-to-stock marketing strategy.

3. High number of bill-of-materials levels.

4. Low average number of parts, etc. per bill-of-material level.

A small-engine assembly plant is an example of an MRP cost-effective context. The plant is characterized as continuous production to stock with an average of five bill-of-material levels (classified as high) and about five components per bill-of-material level (classified as low) and has a B MRP system.

These cost-effective characterizations are based upon data depicting 
Table 2. Logistic regression for MRP use $=B$ or $A$

\begin{tabular}{lccccc}
\hline Function = LOGISTIC & 52 cases & 4 iterations \\
\multicolumn{5}{c}{ Model } \\
& Marginal & Full & Diff \\
-2log (Likelihood) & 65.726 & 62.190 & 3.5358 & df $=4$ & sig $=0.4725$ \\
Fraction explained & 0.5315 & 0.5499 & 0.0184
\end{tabular}

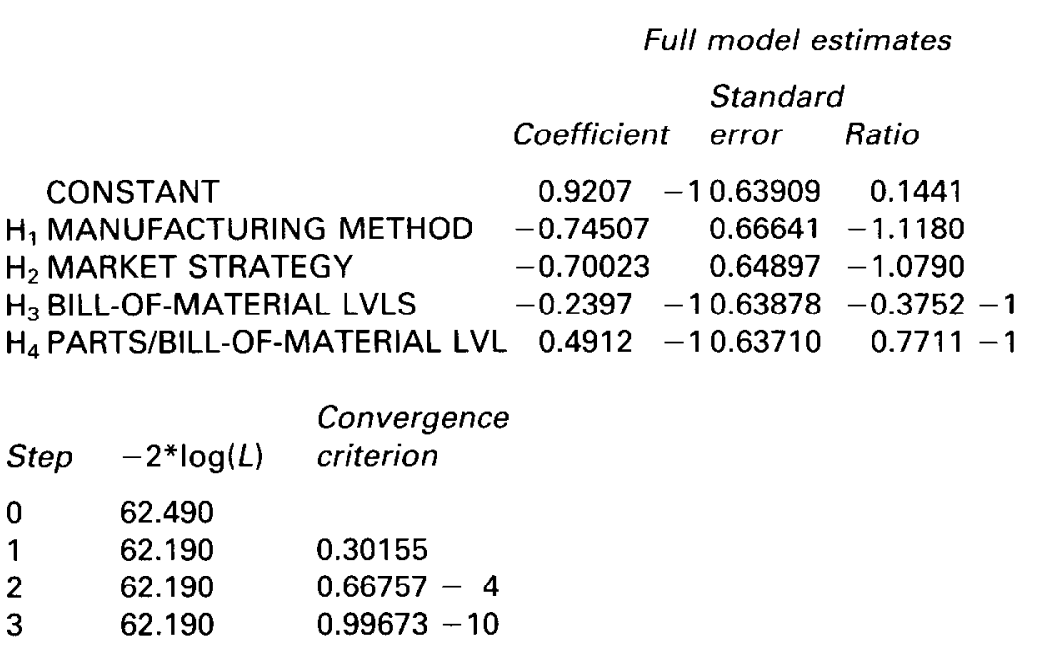

the successful use or non-use of MRP. It would be interesting, in addition, to determine whether the proposed reciprocal MRP-ReorderPoint relationship exists. That is, does the logistic model hold when comparing MRP use to Reorder-Point use? To answer this question, D MRP users are eliminated from the sample, leaving Reorder-Point users coded as 1 and MRP $\mathrm{C}$ and above users coded as 2 . The highly significant (alpha $=0.0065$ ) results are illustrated in Table 3 ; each hypothesis continues to be significant (alpha less than or equal to 0.11). Thus, Reorder-Point MIS tends to be used in the hypothesized manufacturing contexts, which are characterized by:

1. Intermittent (job shop) manufacturing method.

2. Make-to-order marketing strategy.

3. Low number of bill-of-material levels.

4. High average number of parts, etc. per bill-of-material level.

A pump manufacturer is an example of a Reorder-Point cost-effective context. The firm manufactures 90 per cent to order in a job shop with an average of four bill-of-material levels (classified as low) and about 33 parts, etc. per bill-of-material level (classified as high) and has a Reorder-Point system.

\section{Discussion}

This section describes the last cost analysis step: conclusions concerning the cost-effectiveness of the alternative MIS.

Although depicting current MRP use, the logit models can be interpreted in light of the cost analysis hypotheses. That is, given the microeconomic argument of rational management trade-offs or the 
Table 3. Logistic regression for MRP versus Reorder-Point

\begin{tabular}{llllll}
\hline Function = LOGISTIC & 49 cases & 4 iterations \\
\multicolumn{5}{c}{ Model } \\
& Marginal & Full & Diff \\
-2log (Likelihood) & 58.630 & 44.353 & 14.227 & df $=4$ & sig $=0.0065$ \\
Fraction explained & 0.5498 & 0.6360 & 0.0862 &
\end{tabular}

\begin{tabular}{|c|c|c|c|c|c|c|}
\hline & \multicolumn{4}{|c|}{ Full model estimates } \\
\hline & & & Coefficient & $\begin{array}{l}\text { Standard } \\
\text { error }\end{array}$ & Ratio & $\begin{array}{l}\text { 1-tailed } \\
\text { alpha }\end{array}$ \\
\hline $\begin{array}{l}\mathrm{CC} \\
\mathrm{H}_{1} \mathrm{M} \\
\mathrm{H}_{2} \mathrm{M} \\
\mathrm{H}_{3} \mathrm{Bl} \\
\mathrm{H}_{4} \mathrm{PA}\end{array}$ & $\begin{array}{l}\text { JSTANT } \\
\text { VUFACTUR } \\
\text { RKET STRA } \\
\text {-OF-MATEF } \\
\text { TS/BILL-OF }\end{array}$ & $\begin{array}{l}\text { JG METHOD } \\
\text { EGY } \\
\text { AL LVLS } \\
\text { MATERIAL LV }\end{array}$ & $\begin{array}{c}-3.2246 \\
-2.0367 \\
-0.95313 \\
1.1344 \\
-2.1441\end{array}$ & $\begin{array}{l}1.0508 \\
0.89625 \\
0.77520 \\
0.85850 \\
0.92207\end{array}$ & $\begin{array}{r}-3.0687 \\
-2.2725 \\
-1.2295 \\
1.3214 \\
-2.3253\end{array}$ & $\begin{array}{l}0.00 \\
0.01 \\
0.11 \\
0.10 \\
0.01\end{array}$ \\
\hline Step & $-2^{*} \log (L)$ & $\begin{array}{l}\text { Convergen } \\
\text { criterion }\end{array}$ & & & & \\
\hline 0 & 45.478 & & & & & \\
\hline 1 & 44.378 & 1.0231 & & & & \\
\hline 2 & 44.353 & $0.24211-$ & & & & \\
\hline 3 & 44.353 & $0.36613-$ & & & & \\
\hline 4 & 44.353 & $0.88687-1$ & & & & \\
\hline
\end{tabular}

contingency theoretic competitive advantage argument, the use of MRP in specific contexts can be reinterpreted as indicating MRP costeffectiveness in those contexts. Based upon this rationale, the failure of many MRP systems may be due to attempts to install MRP in inappropriate (cost-ineffective) manufacturing contexts, such as intermittent instead of continuous or make-to-order rather than make-tostock. Interestingly, historical suggestions for MRP implementation are typically in such cost-ineffective contexts. (For example, Orlicky ${ }^{24}$ suggests MRP for complex job shop contexts. Only the high number of bill-of-material levels, identified as appropriate here, are in accord with historical guidance.)

Although this discrepancy may initially be unsettling, it is intuitively appealing. When compared to Reorder-Point, MRP is very costly to implement and maintain, and has a dependent structure which is prone to compounding errors. (Due to MRP's bill-of-material cxplosion approach, errors are compounded as requirements are determined for successively lower bill-of-material levels.) In addition, MRP requirements are typically determined using local optimizing algorithms at each bill-of-material level; this simple structure rarely reflects the actual production context. On the other hand, Reorder-Point estimates are independent, reducing the impact of the erroneous estimation of one material requirement upon the estimations of other material requirements. Thus, when the context accurately reflects the MRP model, MRP use can reap substantial benefits compared to Reorder-Point use. However, Reorder-Point seems to be more robust in contexts which are more complex, and not totally in accord with those required by MRP's

${ }^{24}$ ORI.IKKY, op. cit., Ref 17

${ }^{25}$ Op. cit., Ref 10. relatively simplistic model. This finding is in accord with Morecroft $\mathrm{s}^{25}$ simulation comparison of MRP and Reorder-Point systems. 
The findings of this study can be used by MIS designers (both users and analysts) to help develop appropriate manufacturing information systems. A designer would first identify where the firm stands in terms of the four contextual dimension measures. (The manufacturing method, marketing strategy, etc. are easily determined in most firms.) The most clear-cut cases are those that meet all of the MRP or Reorder-Point cost-effectiveness conditions. However, the logit model provides the following help in other cases. Although the model coefficients are not directly interpretable, as are linear regression coefficients, they can give information about relative impacts. For example, referring to Table 3 , differences in manufacturing method or differences in parts per bill-of-material level are expected to have a more significant impact than differences in marketing strategy or bill-of-material levels. Thus, a firm which is characterized in terms of all MRP cost-effective contexts except marketing strategy would be more likely to support MRP than a firm characterized in terms of all MRP cost-effective contexts except manufacturing method.

These relationships are demonstrated in Figure 4. Employing the logit model in Table 1, this figure illustrates the probabilitics of MRP use in various manufacturing contexts. For example, the small-engine assembly plant described above as continuous production to stock with high average bill-of-material levels and low components per bill-of-material level is depicted as having a 99 per cent chance of using MRP; the pump manufacturer characterized as intermittent production to order with low average bill-of-material levels and high components per bill-of-material level is depicted as having a 1 per cent chance of using MRP. Although illustrating probabilities of current MRP use, Figure 4 can be reinterpreted in light of the cost analysis hypotheses as probabilities of MRP cost-effectiveness. These probabilities can thus be used by MIS designers to help evaluate the appropriateness of implementing MRP in contexts which are not clearly MRP cost-effective or cost-ineffective.

Another finding is of interest to information system designers. Manufacturing contexts did not seem to affect the transition from $\mathrm{C}$ MRP systems to A or B MRP systems. This may be due to a threshold effect, where specific contextual conditions must be met before MRP of any type is cost-effective. After this threshold has been met, the effectiveness of MRP improvements may be contingent upon other factors, such as resistance to change, or management's lack of commitment to MRP, which were mentioned at the beginning of this paper.

In addition to aiding information system designers, information

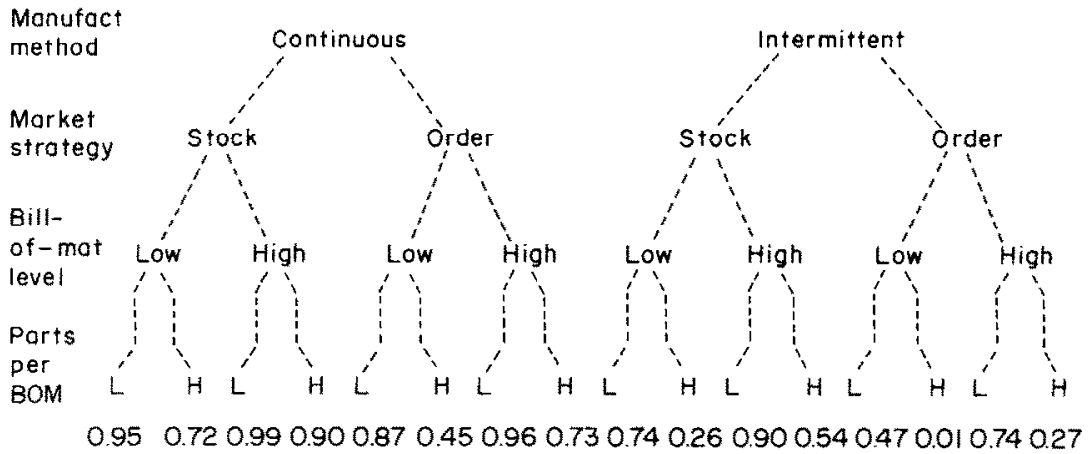

Figure 4. Probabilities of MRP use/cost-effectiveness 
resource planners (including users) can use the MRP versus ReorderPoint information to help plan future MIS requirements. For example, Hayes and Wheelwright ${ }^{26}$ describe typical manufacturing contexts associated with the product life cycle. The product life cycle is divided into four stages, starting with low volume, custom design, one-of-a-kind products and evolving to high volume, high standardization, connodity products. The manufacturing method and marketing strategy associated with these product stages start with intermittent production to order, and end with continuous production to stock. Using the cost analysis hypotheses above, MRP is expected to be more cost-effective later in the product lifecycle, and Reorder-Point more cost-effective carlier in the product lifecycle. This information can be used to help plan appropriate MIS changes as a firm's product evolves.

\section{Summary}

This paper evaluated alternative manufacturing MISs contingent upon various manufacturing contexts. This evaluation can help system analysts and users develop appropriate manufacturing MISs by allowing them to choose the most cost-effective MIS for their firm's current context. Similarly, this evaluation can help information resource managers and users plan future manufacturing MIS requirements, by enabling them to match costeffective MIS to the firm's changing context.

Cost analysis was introduced as the evaluation vehicle used within a contingency theoretic framework. Details surrounding this evaluation methodology were included, in part, to provide a theoretical basis for reinterpreting $M R P$ use data as MRP cost-effectiveness data. The cost analysis-contingency theory synthesis facilitated the choice of relevant MIS and contextual dimensions, and gave a framework for analysing and evaluating their interaction. From this guidance, hypotheses concerning the cost-effectiveness of the alternative MIS were developed, tested and interpreted. This example illustrates the potential for using a microeconomic cost-curve-based analysis without the direct

2"HAYIS, R.II. ANI) WIHIII,WRICHHT. S.C. (1979). Link manufacturing process and product lifecycles. Haward Business Review. Jamary-February, 1.33-140. estimation of cost parameters. It should be noted, however, that cost analysis also provides a framework for research which identifies actual total cost-curve parameters through, for example, simulation studies. 